\title{
Preise als Geschäftsgeheimnisse nach dem Öffentlichkeitsgesetz
}

Ulysse Tscherrig *

Der vorliegende Beitrag geht der Frage nach, ob Preise als Geschäftsgeheimnisse im Sinne des Öffentlichkeitsgesetzes des Bundes qualifiziert werden können. Hintergrund ist ein Urteil des Bundesgerichts vom 5.Juli 2017. In seinem Urteil 1C_4O/2O17 hat das Bundesgericht die Auffassung der Vorinstanz zu dieser Frage als «jedenfalls vertretbar» bezeichnet (E. 6.2.2). Das Appellationsgericht des Kantons Basel-Stadt hatte Preise in Anwendung des basel-städtischen Rechts als Geschäftsgeheimnisse betrachtet. Weil es um die Anwendung des kantonalen Gesetzes ging, konnte das Bundesgericht nur eine Willkürprüfung vornehmen. Nachfolgend wird der Bundesgerichtsentscheid analysiert und der Frage nachgegangen, wie der Fall im Lichte des Öffentlichkeitsgesetzes des Bundes zu entscheiden wäre.

I. Einleitung. 215

II. Ausgangslage 216

1. Sachverhalt 216

2. Prozessgeschichte 216

3. Bundesgericht 216

III. Die Zugangsverweigerungsgründe im Einzelnen 217

1. Von einem Zugangsverweigerungsgrund zum anderen 217

2. Zugangsverweigerung aufgrund der durch die Behörden zugesicherten Geheimhaltung ..... 218

3. Zugangsverweigerung aufgrund der Beeinträchtigung von Positionen in künftigen Verhandlungen. 220

IV. Zugangsverweigerung aufgrund eines Geschäftsgeheimnisses 221

V. Fazit. 225

Zitiervorschlag: Ulysse Tscherrig, Preise als Geschäftsgeheimnisse nach dem Öffentlichkeitsgesetz, in: sui-generis 2019, S. 214

URL: $\quad$ sui-generis.ch/104

DOI: $\quad$ https://doi.org/10.21257/sg.104

* Ulysse Tscherrig, lic. iur., Rechtsanwalt, Jurist im Rechtsdienst der Schweizerischen Bundeskanzlei (Ulysse.Tscherrig@bk.admin.ch). 


\section{Einleitung}

1 Das Informations- und Datenschutzgesetz vom 9. Juni 2010 des Kantons BaselStadt («IDG/BS») ist seit dem 1. Januar 2012 in Kraft. Das Prinzip des freien Zugangs hat der Gesetzgeber des Kantons Basel-Stadt in § 25 Absatz 1 IDG/BS festgesetzt. Demnach hat jede Person Anspruch auf Zugang zu den bei einem öffentlichen Organ vorhandenen Informationen. Nach § 29 Absatz 1 IDG/BS hat das öffentliche Organ die Bekanntgabe von oder den Zugang zu Informationen im Einzelfall u. a. zu verweigern, wenn ein überwiegendes öffentliches oder privates Interesse entgegensteht. Absatz 2 von $\S 29$ IDG/BS enthält eine nicht abschliessende Aufzählung von öffentlichen und Absatz 3 von privaten Interessen. Im Bundesrecht ist die Aufzählung von Zugangsverweigerungsgründen im Vergleich zur basel-städtischen Regel abschliessend. Nach § 29 Absatz 3 Buchstabe b IDG/BS liegt ein privates Interesse dann vor, wenn durch die Bekanntgabe von oder den Zugang zu Informationen Geschäftsgeheimnisse offenbart würden.

Das am 1. Juli 2006 in Kraft getretene Öffentlichkeitsgesetz des Bundes (BGÖ) ${ }^{1}$ soll die Transparenz über den Auftrag, die Organisation und die Tätigkeit der Bundesverwaltung fördern; zu diesem Zweck trägt es zur Information der Öffentlichkeit bei, indem es den Zugang zu amtlichen Dokumenten gewährleistet (Art. 1 BGÖ). Ob ein solcher Zugang zu amtlichen Dokumenten zu gewähren ist, überprüft die Bundesverwaltung auf $\mathrm{Ge}$ such hin (passive Information). Grund-

Bundesgesetz vom 17. Dezember 2004 über das Öffentlichkeitsprinzip der Verwaltung (Öffentlichkeitsgesetz, BGÖ; SR 152.3). sätzlich regelt das Öffentlichkeitsgesetz ausschliesslich die passive Information und verpflichtet nicht zur Veröffentlichung bestimmter amtlicher Dokumente ${ }^{2} .3$ Die Pflicht zur aktiven Information ergibt sich gestützt auf Artikel 180 Absatz 2 der Bundesverfassung, aus Artikel 10 RVOG4 sowie aufgrund des Publikationsrechts des Bundes (PublG5, PublV6).

3 Der Bundesgesetzgeber hat das Öffentlichkeitsprinzip in Artikel 6 Absatz 1 BGÖ festgesetzt. Demnach hat jede Person das Recht, amtliche Dokumente einzusehen und von den Behörden Auskünfte über den Inhalt amtlicher Dokumente zu erhalten. Damit hat der Gesetzgeber die Vermutung des freien Zugangs zu amtlichen Dokumenten eingeführt, womit die Behörde allfällige Ausnahmen vom Öffentlichkeitsprinzip belegen muss und die Beweislast bei der Behörde liegt.7 Solche Regeln, aufgrund derer bestimmte Informationen des Bundes nicht oder noch nicht bzw. nur teilweise zugänglich gemacht werden müssen, finden sich in

2 Stephan C. Brunner, in Brunner/Mader (Hrsg.), Stämpflis Handkommentar zum BGÖ, Art. 1, Rz. 12 f.

3 Eine Ausnahme sieht Artikel 19 der Verordnung vom 24. Mai 2006 über das Öffentlichkeitsprinzip der Verwaltung (Öffentlichkeitsverordnung, VBGÖ; SR 152.31) vor, wonach die zuständige Behörde wichtige amtliche Dokumente so schnell wie möglich im Internet verfügbar macht. Die Möglichkeit zum Erlass dieser Bestimmung hat der Gesetzgeber in Art. 21 Bst. c BGÖ geschaffen.

4 Regierungs- und Verwaltungsorganisationsgesetz vom 21. März 1997 (RVOG; SR 172.010).

5 Bundesgesetz vom 18. Juni 2004 über die Sammlung des Bundesrechts und das Bundesblatt (Publikationsgesetz; PublG, SR 170.512).

6 Verordnung vom 7. Oktober 2015 über die Sammlung des Bundesrechts und das Bundesblatt (Publikationsverordnung; PublV, SR 170.512.1).

7 Botschaft vom 12. Februar 2003 zum Bundesgesetz über die Öffentlichkeit der Verwaltung (Öffentlichkeitsgesetz, BGÖ), BBl 2003 1963, Ziff. 2.2.1.1. 
Artikel 7 BGÖ (Ausnahmen), Artikel 8 BGÖ (Besondere Fälle) und Artikel 9 BGÖ (Schutz von Personendaten).

Weil der Bundesgesetzgeber in Artikel 7 Absatz 1 BGÖ die Geheimhaltungsgründe gegenüber dem öffentlichen Interesse am Zugang abschliessend aufführt (vgl. Rz. 1), hat er die Interessenabwägung bereits vorweggenommen. So ist in der bundesrechtlichen Regelung der Zugang zu amtlichen Dokumenten zu verweigern, wenn dadurch Geschäftsgeheimnisse offenbart werden können (Art. 7 Abs. 1 Bst. g BGÖ). Muss die Bundesbehörde von einem ernsthaften Risiko des Eintritts eines substanziellen Schadens ausgehen, ist das Dokument - ohne Rücksichtnahme auf die Gründe für einen Zugang - nicht zugänglich. ${ }^{8}$ Damit findet auf Bundesebene keine Abwägung zwischen dem privaten Geheimhaltungsinteresse und dem öffentlichen Interesse an Transparenz statt. Demgegenüber geht das basel-städtische Recht von einer Interessenabwägung aus, verlangt es doch in § 29 Absatz 1 IDG/BS ein überwiegendes öffentliches oder privates Interesse für eine Zugangsverweigerung.

\section{Ausgangslage}

\section{Sachverhalt}

5 Das Konsortium der Schweizer Hochschulbibliotheken, zu welchem auch die Universität Basel gehört, hat unter der Führung der ETH-Bibliothek mit verschiedenen wissenschaftlichen Verlagen, namentlich Elsevier, Springer und Wiley, Lizenzverträge abgeschlossen. Dadurch können die Publikationen dieser Verlage

8 Urs Steimen, in Maurer-Lambrou/Blechta (Hrsg.), BSK BGÖ, Art. 7, Rz. 3. den Nutzenden der verschiedenen Bibliotheken zur Verfügung gestellt werden. Namentlich betreffend Preis wurde für diese Lizenzen zwischen den Verlagen und den Hochschulbibliotheken Vertraulichkeit vereinbart. Mit seinem Gesuch vom 23. Juni 2014 verlangte A. von der Universitätsbibliothek Zugang $\mathrm{zu}$ den Dokumenten (Offerten, Rechnungen, Verträge), aus denen ersichtlich werden soll, wie viel die Universität Basel für Lizenzen an die Verlage Elsevier, Springer und Wiley im Zeitraum von 2010 bis 2016 bezahlt hat oder noch zu bezahlen hat.

\section{Prozessgeschichte}

6 Am 11. August 2014 lehnte die Universitätsbibliothek das Gesuch ab. Dagegen hat A. Rekurs eingelegt, der am 19. Dezember 2014 von der Rekurskommission der Universität Basel ebenfalls abgelehnt wurde. A. zog diesen Entscheid an das Appellationsgericht des Kantons Basel-Stadt weiter. Die Beschwerde wurde am 2. Dezember 2016 abgewiesen und A. erhob am 24. Januar 2017 Beschwerde in öffentlich-rechtlichen Angelegenheiten beim Bundesgericht. A. beantragte, das Urteil sei aufzuheben und die Universität Basel sei zu verpflichten, ihm Einsicht in die Akten zu gewähren, aus denen ersichtlich sei, wie viel die Universität an die Verlage Elsevier, Springer und Wiley im Zeitraum 2010 bis 2016 bezahlt habe; eventualiter sei die Sache zur Ergänzung des Sachverhalts und zu neuer Beurteilung an die Vorinstanz zurückzuweisen.

\section{Bundesgericht}

7 Das Bundesgericht hat die Beschwerde am 5. Juli 2017 abgewiesen. Es begründet seinen Entscheid vornehmlich damit, 
es sei vom Appellationsgericht des Kantons Basel-Stadt «jedenfalls vertretbar, ernsthafte private und öffentliche Interessen von einem gewissen Gewicht für die Verweigerung des Zugangs zur nachgesuchten Information zu bejahen». Die Vorinstanz habe die Lizenzpreise ohne Willkür als überwiegend im Sinne von $\S 29$ Absatz 1 IDG/BS erachten dürften. 9

\section{Die Zugangsverweigerungsgründe im Einzelnen}

\section{Von einem Zugangsverweigerungs- grund zum anderen}

8

Nach § 29 Absatz 1 IDG/BS hat das öffentliche Organ den Zugang zu Informationen im Einzelfall ganz oder teilweise $\mathrm{zu}$ verweigern oder aufzuschieben, wenn eine besondere gesetzliche Geheimhaltungspflicht oder ein überwiegendes öffentliches oder privates Interesse diesem Zugang entgegensteht. Absätze 2 und 3 von $\S 29$ IDG/BS führen solche öffentlichen oder privaten Interessen nicht abschliessend auf. Das Appellationsgericht des Kantons Basel-Stadt verweigerte den Zugang im Wesentlichen aus zwei Gründen: die zugesicherte Geheimhaltung nach § 29 Absatz 3 Buchstabe c IDG/BS und die Beeinträchtigung von Positionen in Verhandlungen nach § 29 Absatz 2 Buchstabe d IDG/BS.

9 In Erwägung 6.2.2. beanstandet das Bundesgericht, der Beschwerdeführer habe sich kaum mit den Erwägungen der Vorinstanz auseinandergesetzt. Das Bundesgericht führt aus, dass jedenfalls die privaten Interessen am Fortbestand der Vertraulichkeit der Lizenzpreise auf

9 Urteil des Bundesgerichts 1C_40/2017 vom 5. Juli 2017 E. 6.2.2. der Hand lägen und das Appellationsgericht diese ohne Willkür, also korrekterweise, als Geschäftsgeheimnis im Sinne von $\S 29$ Absatz 3 Buchstabe b IDG/BS habe ansehen dürfen. Dies aber nur «in Anwendung des basel-städtischen Rechts», jedoch trotz kritischer Beurteilung des EDÖB und trotz abweichenden Entscheiden in anderen Kantonen. Die im basel-städtischen Transparenzrecht verlangte Güterabwägung kann so verstanden werden, dass die zugesicherte Geheimhaltung eines von mehreren Abwägungskriterien gegen den Zugang ist. Anders ist es beim Bund, wo die zugesicherte Geheimhaltung nicht ein Abwägungs-, sondern ein zwingendes Verweigerungskriterium ist. Das lässt den Schluss zu, dass die Anwendung des basel-städtischen Rechts auch zu einem anderen Ergebnis hätte führen können. Denkbar wäre dies, wenn die Interessenabwägung mehr Argumente für statt gegen den Zugang hervorgebracht hätte. Den beiden vom Appellationsgericht ins Feld geführten Zugangsverweigerungsgründen der zugesicherten Geheimhaltung und der Beeinträchtigung von Positionen in Verhandlungen folgend, endet die Begründungslinie des Bundesgerichts damit, dass es zum Schluss kommt, aufgrund der offensichtlichen privaten Interessen an der Vertraulichkeit der Preise liege ein Geschäftsgeheimnis vor. Das Bundesgericht zieht somit infolge der vorgenommenen Interessenabwägung den Schluss, ein Geschäftsgeheimnis läge vor, weil das Konsortium die Geheimhaltung zugesichert hat und weil die Offenlegung der Preise die Positionen des Konsortiums in künftigen Verhandlungen beeinträchtigen könnte. Die Qualifikation des Preises als Geschäftsgeheimnis erfolgt mithin aufgrund von Interessen 
und nicht aufgrund des Wesens des Preises an sich.

Beim Bund hingegen muss die Qualifikation des Preises als Geschäftsgeheimnis isoliert erfolgen. Da das BGÖ keine Interessenabwägung vorsieht, genügt es nach Artikel 7 Absatz 1 Buchstabe g BGÖ, dass ein Schaden entstehen könnte, wenn ein Geschäftsgeheimnis offenbart werden würde. Das Schadenrisiko muss erheblich und ernsthaft sein. Die Ausnahmeklausel als Einschränkung des Zugangsrechts muss daher restriktiv ausgelegt werden und der Schaden muss nach dem gewöhnlichen Lauf der Dinge mit grosser Wahrscheinlichkeit eintreffen. Der Schutz eines Geheimhaltungsinteresses hängt vom Vorhandensein eines Schadenrisikos und nicht von einer Interessenabwägung ab. ${ }^{10}$

Im Bund können nicht verschiedene Ausnahmetatbestände des Öffentlichkeitsprinzips miteinander vermischt werden, um die Durchschlagskraft eines anderen Zugangsverweigerungsgrunds zu untermauern. Auf Bundesebene kann es freilich Fälle geben, in denen verschiedene Zugangsverweigerungsgründe nebeneinander vorliegen. Das gleichzeitige Vorhandensein von zwei oder mehreren verschiedenen Zugangsverweigerungsgründen würde aber nach Öffentlichkeitsrecht des Bundes nicht zu einem weiteren Verweigerungsgrund führen. Die Verweigerungsgründe können im Bund parallel vorliegen, aber nicht «kumuliert» werden. Daher und weil das Öffentlichkeitsrecht des Bundes keine Interessenabwägung kennt, kann der

10 Bertil Cottier, in Brunner/Mader (Hrsg.), Stämpflis Handkommentar zum BGÖ, Art. 7, Rz. 4 f.
Bundesgerichtsentscheid für die Beurteilung von Geschäftsgeheimnissen nach dem Öffentlichkeitsgesetz des Bundes keine präjudizielle Wirkung entfalten.

\section{Zugangsverweigerung aufgrund der durch die Behörden zugesicherten Geheimhaltung}

12 Nach Artikel 7 Absatz 1 Buchstabe h BGÖ wird der Zugang zu amtlichen Dokumenten eingeschränkt, aufgeschoben oder verweigert, wenn durch seine Gewährung Informationen vermittelt werden können, die der Behörde von Dritten freiwillig mitgeteilt worden sind und deren Geheimhaltung die Behörde zugesichert hat. Die seitens der Verwaltung gegenüber Dritten erfolgte Zusicherung der Geheimhaltung ist wie im IDG/BS ein Zugangsverweigerungsgrund. Im Bund ist sie ein absoluter Verweigerungsgrund, in Basel eines von mehreren Abwägungskriterien.

13 Für die Anrufung dieser Ausnahme vom Öffentlichkeitsprinzip des Bundes müssen nach der Lehre ${ }^{11}$ kumulativ drei Voraussetzungen erfüllt sein. Erstens muss die Information von einer Privatperson stammen. Dieses Kriterium wäre vorliegend erfüllt, stammen doch die Informationen von den privaten Verlagen. Die zweite Voraussetzung dieser Ausnahme des Öffentlichkeitsprinzips ist, dass die Information der Behörde freiwillig mitgeteilt worden ist. Auch dies wäre vorliegend erfüllt, weil die Geschäftsverhältnisse zwischen der Universität Basel und den Verlagshäusern auf vertraglicher Basis ${ }^{12}$ beruhen. Ein Vertrag ist eine gegen-

11 Bertil Cottier (Fn. 10), Art. 7, Rz. 47.

12 Informationen, welche Dritte der Behörde aufgrund vertraglicher Verpflichtungen mitgeteilt haben, werden nicht «von sich aus» und damit 
seitig übereinstimmende Willensäusserung (vgl. Art. 1 Abs. 1 OR), dessen Abschluss freiwillig erfolgt. Die Pflicht zur Erfüllung des Vertrags und damit vorliegend die Lieferung der Information ergibt sich aus dem Vertrag. Diesen Vertrag haben die Parteien aus freiem Willen - also freiwillig - abgeschlossen. Die dritte und letzte Voraussetzung ist die Zusicherung der Geheimhaltung der Behörde gegenüber den Dritten. Diese Voraussetzung im Recht des Bundes entspricht derjenigen von § 29 Absatz 3 Buchstabe c IDG/BS. Auch dieses Kriterium der Zusicherung ist vorliegend gemäss Bundesgerichtsentscheid erfüllt ${ }^{13}$. Ebenso gilt hier, dass die zugesicherte Geheimhaltung im basel-städtischen Recht als Abwägungskriterium und im Bundesrecht als absoluter Ausnahmegrund gegen den Zugang zählt (vgl. Rz. 9).

nicht freiwillig mitgeteilt. Nach der hier vertretenen Meinung ist eine vertragliche Pflicht eine «freiwillige Pflicht». Ein Vertrag ist eine Abmachung, mittels welcher sich die Parteien freiwillig $\mathrm{zu}$ etwas verpflichten. Diese Pflicht ist nicht gleichzusetzen mit einer gesetzlichen Pflicht. Den Abschluss des Vertrags und damit das daraus resultierende Vertragsverhältnis gehen die Vertragsparteien freiwillig ein. Nach Art. 1 Abs. 1 OR ist «zum Abschluss eines Vertrages die übereinstimmende gegenseitige Willensäusserung der Parteien erforderlich». Es besteht eine Pflicht zur Vertragseinhaltung («pacta sunt servanda»). Es gibt aber keine Pflicht zum Abschluss eines Vertrages, da dieser freiwillig erfolgt. Cottier erwähnt a. a. $\mathrm{O}$ «gesetzliche Verpflichtungen», wogegen nichts einzuwenden ist, zumal im Verhältnis zwischen Staat und Bürger der öffentlich-rechtliche Grundsatz der Unterordnung und nicht wie im Privatrecht der Grundsatz der Privatautonomie gilt. Näheres zum rechtsgeschäftlichen Willen (auch Gestaltungswillen), v. a. die Unterteilung des Willens in drei Elemente: Christoph Müller, Berner Kommentar zum OR, 2018, Rz. 15 f. zu Art. 1.

13 Vgl. Urteil des Bundesgerichts 1C_40/2017 vom 5. Juli 2017: Sachverhalt A. «Namentlich hinsichtlich des Preises wurde zwischen den Verlagen und Hochschulbibliotheken Vertraulichkeit vereinbart.»
14 Betreffend die seitens der Verwaltung gegenüber Dritten zugesicherte Vertraulichkeit des Lizenzpreises zieht das Bundesgericht den Schluss, dass die zugesicherte Vertraulichkeit als Geschäftsgeheimnis zu qualifizieren ist, indem es in seiner Begründung ausführt, die Vorinstanz habe die Vertraulichkeit in Anwendung des basel-städtischen Rechts «trotz kritischer Einschätzung des Eidgenössischen Datenschutz- und Öffentlichkeitsbeauftragten und abweichenden Entscheiden in andern Kantonen» 14 ohne Willkür als Geschäftsgeheimnisse im Sinne von § 29 Absatz 3 Buchstabe b IDG/BS ansehen dürfen. Das Bundesgericht folgert, die zugesicherte Vertraulichkeit dürfe als Geschäftsgeheimnis angesehen werden. Dies mag aufgrund der erfolgten Willkürprüfung und damit «in Anwendung des basel-städtischen Rechts» stimmen (vgl. Rz. 9). Auf Bundesebene kann eine Information durch die Tatsache der zugesicherten Vertraulichkeit jedoch nicht zu einem Geschäftsgeheimnis werden. Im Bundesrecht ist der Inhalt der fraglichen Informationen allein - vorliegend die vereinbarten Preise - massgebend, ob ein Geschäftsgeheimnis vorliegt oder nicht.

15 Der Logik des Bundesgerichtes folgend könnte nach basel-städtischem Recht jede Information durch die Tatsache der Zusicherung der Vertraulichkeit zum Geschäftsgeheimnis werden. Für die Verwaltung des Kantons Basel-Stadt wäre es ein Leichtes, Informationen durch eine Vertraulichkeitszusicherung $\mathrm{zu}$ einem Geschäftsgeheimnis zu machen. Eine analoge Auslegung auf Bundesebene

14 Urteil des Bundesgerichts $1 \mathrm{C} \_40 / 2017$ vom 5. Juli 2017 E. 6.2.2., Seite 10. 
würde das Öffentlichkeitsprinzip seines Gehaltes entleeren.

Nach Artikel 7 Absatz 1 Buchstabe h BGÖ ist die zugesicherte Geheimhaltung zwar auch im Bund ein Grund, anbegehrte Informationen nicht zugänglich machen $\mathrm{zu}$ müssen. Anknüpfungspunkt dieser Ausnahmebestimmung ist die Zusicherung der Vertraulichkeit seitens der Behörde - neben den erwähnten beiden Voraussetzungen, dass die Information von einer Privatperson stammt und die Mitteilung freiwillig erfolgte (vgl. oben RZ 12 ff.). Die Zusicherung der Vertraulichkeit kann aber losgelöst vom Inhalt der Information erfolgen. Dabei kann der Inhalt der Information durchaus ein Geschäftsgeheimnis sein. Dann müsste aber keine Zusicherung der Vertraulichkeit mehr erfolgen, weil die Geschäftsgeheimnisse unter eine andere Ausnahmebestimmung des Öffentlichkeitsprinzips fallen würden (Art. 7 Abs. 1 Bst. g BGÖ). Aus der Begründung des Urteils kann geschlossen werden, dass die Ausnahmebestimmung der zugesicherten Vertraulichkeit abgesondert für die Zugangsverweigerung nicht reichen würde, im baselstädtischen Recht jedoch als Abwägungskriterium gegen den Zugang beigezogen wird. Eine zu weit gehende Auslegung dieser Ausnahmebestimmung könnte dazu führen, dass die Zugänglichkeit letztlich vom Willen eines Dritten abhängig wäre, womit das Öffentlichkeitsprinzip sowohl in Basel als auch im Bund seines Sinnes entleert werden würde ${ }^{15}$. Das dürfte mit ein Grund dafür sein, warum das Bundesgericht zur Festigung seiner Argumentation neben der zugesicherten Vertraulichkeit noch weitere Zugangsverweigerungsgründe erwogen hat.

15 Bertil Cottier (Fn.10), Art. 7, Rz. 46.

\section{Zugangsverweigerung aufgrund der Beeinträchtigung von Positio- nen in künftigen Verhandlungen}

17 In Erwägung 6.2.1. setzt sich das Bundesgericht mit §29 Absatz 2 Buchtabe d IDG/BS und der Argumentation des Appellationsgerichts des Kantons BaselStadt dazu auseinander. Demnach sei namentlich dann ein öffentliches $\mathrm{Ge}$ heimhaltungsinteresse gegeben, wenn die Position in Verhandlungen beeinträchtigt würde. Dies liege hier vor, weil die Verlage die bisherigen Lizenzpreise im Vertrauen auf vertragliche Geheimhaltungsklauseln gewährt hätten. Bei einer Bekanntgabe dieser Informationen könnten Dritte diese verwenden und damit versuchen, die Preise zu drücken. So könnte es sein, dass dem Konsortium der Schweizer Hochschulbibliotheken nicht mehr Preisreduktionen im gleichen Umfang gewährt werden würden. Dies würde letztlich wohl zu höheren, nicht zu tieferen Beschaffungskosten führen.

18 Die Annahme, dass künftige Preise aufgrund zugänglich gemachter Preise steigen könnten, ist unter dem Aspekt der Beeinträchtigung von Positionen in künftigen Verhandlungen ein ökonomisches und hypothetisches, aber kein rechtliches Argument. Gesamtwirtschaftliche Gründe bezogen auf den Preis sind als Ausnahme zum Transparenzgrundsatz weder im Recht des Kantons Basel-Stadt noch dem des Bundes vorgesehen. Daher kann es offenbleiben, ob der Zugang tatsächlich höhere Beschaffungskosten zur Folge hätte.

19 Auch im Bundesrecht sind amtliche Dokumente über Positionen in laufenden und künftigen Verhandlungen nicht zugänglich und zwar «in keinem Fall» 
(Art. 8 Abs. 4 BGÖ). Es handelt sich hierbei um einen absoluten Schutz, womit auch hier eine Abwägung ausgeschlossen ist. Das Bundesgericht reichert dieses Argument in Anwendung des basel-städtischen Rechts indessen mit den möglicherweise steigenden Preisen und den im Vorfeld vertraglich zugesicherten Geheimhaltungsklauseln an und verwebt dadurch verschiedene Zugangsverweigerungsgründe. Der eine Punkt ist ein ökonomischer und hat nur mutmassenden Charakter. Der andere betrifft die oben behandelte Ausnahme der erfolgten $\mathrm{Zu}$ sicherung (Rz. $12 \mathrm{ff}$.). Wie dargelegt ist diese Interessenabwägung im baselstädtischen Recht vorgesehen, im Bundesrecht jedoch nicht.

\section{Zugangsverweigerung aufgrund eines Geschäftsgeheimnisses}

In Erwägung 6.2.2. führt das Bundesgericht aus, die privaten Interessen an der Vertraulichkeit der Lizenzpreise seien offensichtlich, und verweist dabei auf die Lehre $^{16}$, welche die Geschäftsstrategie und die Preiskalkulation als Geschäftsgeheimnisse betrachtet. Das private Interesse allein ist aber sowohl im baselstädtischen als auch im Schweizer Recht nicht ausreichend, damit eine Information als Geschäftsgeheimnis qualifiziert werden kann. In Basel-Stadt sind die privaten Interessen Abwägungskriterien gegen den Zugang. Im Bund hingegen

16 Beat Rudin in: Praxiskommentar zum Informations- und Datenschutzgesetz des Kantons BaselStadt, Beat Rudin/Bruno Baeriswyl (Hrsg.), 2014 (zit. Praxiskommentar) $\S 29$, Rz. 47 ff., der in Rz. 48 ausdrücklich die Geschäftsstrategie und die Preiskalkulation als Geschäftsgeheimnisse anspricht; ebenso für das Öffentlichkeitsgesetz des Bundes, Cottier/Schweizer/Widmer, in: Öffentlichkeitsgesetz, Stephan C. Brunner/Luzius Mader (Hrsg.), 2008, Art. 7 N. 43. sind die privaten Interessen als Kriterien für die Qualifikation einer Information als Geschäftsgeheimnis beizuziehen.

21 Der Begriff des Geschäftsgeheimnisses ist gesetzlich nicht definiert'17. Als Geheimnis gelten nach ständiger Praxis des Bundesgerichts weder offenkundige noch allgemein zugängliche Tatsachen (relative Unbekanntheit), die ein Geheimnisherr berechtigterweise geheim halten möchte (subjektiver Geheimhaltungswille) und an deren Geheimhaltung der Geheimnisherr ein objektiv berechtigtes Geheimhaltungsinteresse (objektives Geheimhaltungsinteresse) hat ${ }^{18}$. Gegebenheiten wie Marktanteile eines einzelnen Unternehmens, Umsätze, Preiskalkulationen, Rabatte und Prämien, Bezugs- und Absatzquellen, interne Organisation eines Unternehmens, Geschäftsstrategien und Businesspläne sowie Kundenlisten und beziehungen weisen in der Regel ein $o b$ jektives Geheimhaltungsinteresse auf. 19

22 Unter Geschäftsgeheimnisse fallen diejenigen Informationen, deren Kenntnisnahme dazu führen würde, dass dem betroffenen Unternehmen ein Wettbewerbsvorteil genommen wird und diese Kenntnisnahme Wettbewerbsverzerrungen zur Folge hätte. ${ }^{20}$ Artikel 7 Absatz 1 Buchstabe g BGÖ ist nur auf Geschäftsinformationen anwendbar, deren Kenntnisnahme durch die Konkurrenz zu Marktverzerrungen führen würde oder wodurch dem betroffenen Unternehmen ein Wettbewerbsvorteil genommen bzw. ein Wettbewerbsnachteil verschafft

17 Urteil des Bundesgerichts 1C_665/2017 vom 16. Januar 2019.

18 BGE 142 II 268 E. 5.2.2.1 S. 276.

19 Urteil des Bundesverwaltungsgerichts A-6108/2016 vom 28. März 2018 E. 6.1.2.

20 Bertil Cottier (Fn. 10), Art. 7, Rz. 41. 
wird. ${ }^{21}$ Das Bundesgericht hatte im vorliegenden Entscheid aber nicht Bundesrecht, sondern die korrekte Anwendung kantonalen Rechts zu beurteilen. Daher legt das Bundesgericht in seinen Erwägungen nicht dar, welche wirtschaftlichen Schäden dem Konsortium drohen würde und inwiefern der Zugang zu den Preisen konkret zu Wettbewerbsnachteilen des Konsortiums beziehungsweise zu Wettbewerbsvorteilen der Konkurrenz führen würde.

Auch der Bundesrat wollte mit dem Entwurf zum BGÖ eine Verzerrung des Wettbewerbs zwischen den Marktteilnehmenden durch die Einführung des Öffentlichkeitsprinzips verhindern, weshalb er dem Parlament mit den Geschäftsgeheimnissen eine Ausnahme vom Öffentlichkeitsprinzip vorgeschlagen hatte..22 Gegenstand des Geschäftsgeheimnisses müssen geschäftlich relevante Informationen betreffen; dazu gehören unter anderem Informationen über Einkaufsund Bezugsquellen, zur Betriebsorganisation und zur Preiskalkulation. Des Weiteren sind Geschäftsstrategien Informationen, deren Gehalt geschäftsrelevant ist; auch Businesspläne sowie Kundenlisten und -beziehungen fallen unter Geschäftsgeheimnisse. Diese Informationen haben einen betriebswirtschaftlichen oder kaufmännischen Charakter. Massgebend ist, ob die Informationen Auswirkungen auf das Geschäftsergebnis haben können, bzw. ob die Informationen Auswirkungen auf die Wettbewerbsfähigkeit der Unternehmung haben. ${ }^{23}$ Geschäftsgeheimnisse

21 Empfehlung EDÖB vom 24. Februar 2017, E. 13.

22 Botschaft zum Bundesgesetz über die Öffentlichkeit der Verwaltung (Öffentlichkeitsgesetz, BGÖ vom 12. Februar 2003 [BBl 2003 1963]), S. 2012.

23 Urteil des Bundesgerichts 1C_665/2017 vom 16. Januar 2019 E. 3.3.; Bertil Cottier (Fn. 10), müssen von Bedeutung sein für den wirtschaftlichen Erfolg und damit von wirtschaftlichem Wert sein. ${ }^{24}$ Als Geschäftsgeheimnisse kommen ferner alle Tatsachen technischer, organisatorischer, kommerzieller und finanzieller Natur des wirtschaftlichen Lebens infrage, soweit diese Tatsachen Einfluss auf den wirtschaftlichen Erfolg des Geheimnisherrn haben könnten. ${ }^{25}$ Für einen Verlag können beispielsweise die spezifischen Druckverfahren ein Geschäftsgeheimnis sein. Für eine Unternehmung in der Lebensmittelbranche kann ein Rezept ein Geschäftsgeheimnis sein; dies freilich nicht in Bezug auf die auf der Verpackung angegebenen Zutaten, aber doch zumindest in Bezug auf die Mengenverhältnisse der Zutaten.

24 Auch nach der hier vertretenen Meinung kann die Preiskalkulation an sich oder zumindest Teile davon - Kriterien und Informationen, die zu einem Preis führen - durchaus ein Geschäftsgeheimnis sein. Der Preis als Resultat dieser Preiskalkulation ist von der Kalkulation an sich zu unterscheiden und stellt eine andere Information dar. Die Preiskalkulation ist ein Vorgang und der Preis ist das Resultat dieses Vorgangs. Ein Entscheidfindungsprozess ist nicht gleichzusetzen mit dem aus diesem Prozess hervorgehenden Entscheid. Auch das Planen ist von dessen Resultat - dem Plan - zu unterscheiden. Mit der Aussage, die privaten Interessen am Fortbestand der Vertraulichkeit der Lizenzpreise lägen auf der Hand und daher seien diese Lizenzpreise als

Art. 7, Rz. 43.

24 Stefan Trechsel/ Marc Jean-Richard-dit-Bressel, Schweizerisches Strafgesetzbuch, Praxiskommentar, 2013, Rz. 6 zu Art. 162.

25 Urteil des Bundesverwaltungsgerichts A-4571/2015 vom 10. August 2016 E. 6.3. 
Geschäftsgeheimnisse im Sinne von $\S 29$ Absatz 3 Buchstabe b IDG/BS anzusehen, stützt sich das Bundesgericht primär auf den dem Dritten zuzuschreibenden subjektiven Willen. Dieser ist jedoch für sich allein betrachtet kein Grund, eine Information als Geschäftsgeheimnis zu betrachten - weder im Recht des Kantons Basel-Stadt noch im Bund (vgl. Rz. 22). Dass Preise von mit öffentlichen Geldern finanzierten öffentlich zugänglichen wissenschaftlichen Büchern nicht zugänglich sein sollen und damit die privaten - rein wirtschaftlichen - Interessen höher gewichtet werden als das öffentliche Interesse am $\mathrm{Zu}$ gang zu den Preisen, ist unbefriedigend.

Entscheidend ist, ob der Zugang zu den Lizenzpreisen und ihre potenzielle Veröffentlichung die Wettbewerbsfähigkeit der Verlage beeinträchtigen könnten. Dazu müssten die Lizenzpreise, um als Geschäftsgeheimnisse infrage $\mathrm{zu}$ kommen, für den geschäftlichen Erfolg der Verlage von Bedeutung sein. Das wird weder vom Appellationsgericht des Kantons Basel-Stadt noch vom Bundesgericht dargelegt. Das Bundesgericht zeigt indes auch nicht auf, inwiefern die Offenlegung der verlangten Lizenzpreise den weiteren Geschäftsgang der Verlage derart zu beeinflussen vermögen würde, dass dadurch die Wettbewerbsfähigkeit der Verlage beeinträchtigt werden könnte.

Beschaffungen der öffentlichen Hand, die mit öffentlichen Geldern und damit durch die Steuerzahlenden finanziert werden, unterliegen grundsätzlich dem Öffentlichkeitsprinzip des Bundes. Es sollte selbstverständlich sein, dass somit auch die Höhe der Preise der Beschaffungen transparent sein müsste. Mit dem
Sinn und Zweck des Öffentlichkeitsprinzips des Bundes nicht vereinbar sein kann, dass allein die bezahlten Preise nicht zugänglich sein sollen, während gleichzeitig öffentlich bekannt ist, welche Objekte öffentlich beschafft werden. Vorliegend sind das die Bücher. Auch in anderen Bereichen ist öffentlich bekannt, welche Objekte die öffentliche Hand erworben hat. Dazu gehört auch die Angabe der Preise.

27 Das Bundesgericht hat die Qualifikation der Preise als Geschäftsgeheimnis nach basel-städtischem Recht vorliegend ohne weitere Analyse akzeptiert. Dies ist auf die eingeschränkte Kognition des Bundesgerichts zurückzuführen. Es gab sich aber mit der Begründung, Preise seien für sich allein Geschäftsgeheimnisse, auch nicht zufrieden, weshalb es noch weitere Ausnahmegründe erwogen hat. Dabei bleibt einzig die Ausnahme der zugesicherten Geheimhaltung überzeugend. Dies aber nur, weil die Verlage die Verträge im Vertrauen auf diese zugesicherte Geheimhaltung abgeschlossen haben. Wie erwähnt, darf die Möglichkeit der Zusicherung von Geheimhaltung nicht dazu führen, dass die Beurteilung über die Einschränkung des Öffentlichkeitsprinzips in der Hand Dritter liegt (vgl. Rz. 16). Zudem birgt diese Möglichkeit seitens der Verwaltung ein gewisses Missbrauchspotenzial. Die Verwaltung könnte in Versuchung geraten, Vertraulichkeitszusicherungen Dritten gegenüber standardmässig vorzuschlagen.

28 Gegen den Zugang zu einem Preis könnte ins Feld geführt werden, die Kenntnis über den Preis lasse Rückschlüsse auf 
Rabattgewährung $\mathrm{zu}^{26}$. Einzig wenn dargelegt werden könnte, dass auf diesem Umweg etwas über die Kalkulation der Preise oder zumindest die Rabattstrategie in Erfahrung gebracht werden kann, würde das für eine Qualifikation des Preises als Geschäftsgeheimnis sprechen. Bei Endpreisen dürfte das aber ausgeschlossen sein. Aus der Sicht der Unternehmung stellt sich die Frage, ob der Zugang zum Preis eine finanzielle Beeinträchtigung zur Folge hat; aus der Sichtweise der Konkurrenten ist fraglich, welchen wirtschaftlichen Nutzen andere potenzielle Anbietende aus den bekannten Preisen haben ${ }^{27}$. Dieser Nutzen ist aber nicht massgebend für die Qualifikation als Geschäftsgeheimnis der Preise. Eine weitere Perspektive ist diejenige anderer potenzieller Kunden. Diese könnten allenfalls einen Anspruch auf mindestens gleich hohe wie dem betreffenden Gemeinwesen gewährten Rabatte verlangen. So könnte die Verhandlungsposition des Verlags gegenüber anderen Kunden möglicherweise etwas geschwächt werden. Wenn dem so wäre, könnte tatsächlich die Situation entstehen, dass der Verlag geringere oder keine Rabatte mehr gewähren würde, wenn er weiss, dass der Preis öffentlich wird. Allerdings dürfte dieses Argument wenig überzeugen, wenn der Anbieter ein «Monopolist» oder «Quasi-Monopolist» ist. Davon kann bei den wissenschaftlichen Verlagen - wie vorliegend - ausgegangen werden. Bei anderen Anbietern ohne

$26 \overline{\text { Vgl. Isabelle Häner, in Maurer-Lambrou/Blechta }}$ (Hrsg.), BSK BGÖ, Art. 7, Rz. 40: Verkauf von Liegenschaften durch den Bund: Preise bilden als Teil der Verkaufsbedingungen kein Geschäftsgeheimnis, soweit keine Rückschlüsse auf die Preiskalkulation gezogen werden können.

27 Isabelle Häner, in Maurer-Lambrou/Blechta (Hrsg.), BSK BGÖ, Art. 7, Rz. 36.
Monopolprivileg könnte diese Argumentation durchaus durchdringen, reicht aber für sich allein nicht als Qualifikation von Preisen als Geschäftsgeheimnis.

29 Im öffentlichen Beschaffungsrecht des Bundes besteht eine aktive Informationspflicht seitens des Bundes. Während der Phase des Vergabeverfahrens gilt die Vertraulichkeit betreffend sämtlicher vom Anbieter oder der Anbieterin gemachten Angaben. ${ }^{28}$ Spätestens 30 Tage nach Erteilung des Zuschlags muss die Auftraggeberin Angaben über das Vergabeverfahren veröffentlichen, auch die aus einem freihändigen Verfahren, d. h. ohne öffentliche Ausschreibung. ${ }^{29}$ Die Veröffentlichungspflicht im öffentlichen Beschaffungsrecht des Bundes besteht auch hinsichtlich der Preise des berücksichtigten Angebots (Art. 28 Bst. f VöB). Bezogen auf das Bundesrecht wäre es wohl schwierig zu behaupten, Preise, die nach VöB bekanntzugeben sind, seien als Geschäftsgeheimnisse zu bezeichnen. Das würde bedeuten, dass das öffentliche Beschaffungsrecht des Bundes eine Publikation von Geschäftsgeheimnissen vorsehen würde. Ein derartiger Widerspruch zwischen dem öffentlichen Beschaffungsrecht und dem Öffentlichkeitsprinzip ist nicht gewollt. Ein Preis isoliert betrachtet ist somit kein Geschäftsgeheimnis im Sinne einer Ausnahme des Öffentlichkeitsprinzips des Bundes.

3o Im Privatrechtsverkehr könnten die privaten Rechtssubjekte Preise als Geschäftsgeheimnis betrachten. Das Öffent-

$28 \overline{\text { Art. } 8 \text { Abs. } 1 \text { Bst. d des Bundesgesetzes vom }}$ 16. Dezember 1994 über das öffentliche Beschaffungswesen (BöB; SR 172.056.1).

29 Art. 28 der Verordnung vom 11. Dezember 1995 über das öffentliche Beschaffungswesen (VöB; SR 172.056.11). 
lichkeitsprinzip gilt für die Verwaltung und nicht für den Rechtsverkehr unter Privaten. In den Beziehungen zwischen Privatrechtssubjekten gilt das Öffentlichkeitsprinzip des Bundes nicht. Es kann aber zur Anwendung kommen, sobald Private mit der Verwaltung in Beziehung treten. Aber auch hier kann ein Preis isoliert betrachtet nicht als Geschäftsgeheimnis qualifiziert werden. Ein Preis ist letztlich das Resultat der Vertragsverhandlung zwischen Käufer- und Verkäuferschaft (vgl. Art. 1 Abs. 1 OR; Rz. 13). Als Information ist er somit hälftig sowohl der Sphäre der Käufer- als auch der Verkäuferschaft zuzuordnen. Einzig die Information, wieviel der Bund als Käufer an einen Verkäufer bezahlt hat, muss seitens des Bundes öffentlich zugänglich sein. Die identische Information als Geschäftsgeheimnis zu bezeichnen, nur weil diese der Sphäre der privaten Verkäuferschaft zuzuordnen ist, wäre fehlgeleitet. Weder das oberste Gericht des Kantons Basel-Stadt noch das Bundesgericht haben die Qualifikation von Preisen als Geschäftsgeheimnisse anhand der Kriterien für die Qualifikation von Geschäftsgeheimnissen vorgenommen. Auch in der basel-städtischen Rechtsprechung wurden die Preise nur zusammen mit anderen Abwägungskriterien als Geschäftsgeheimnisse qualifiziert, nicht aber aus sich heraus und isoliert betrachtet.

\section{Fazit}

31 Gemäss Bundesgericht liegen genügend rechtliche Argumente aus dem baselstädtischen Transparenzrecht gegen den Zugang der Listenpreise vor. Das Hauptargument für die Zugangsverweigerung ist die seitens der Behörde gegenüber dem Verlag gemachte Zusicherung der
Geheimhaltung. Ein weiteres Argument gegen den Zugang ist, dass keine Informationen über Positionen in laufenden oder künftigen Verhandlungen offengelegt werden dürfen. Hingegen geht aus dem vorliegenden Bundesgerichtsentscheid nicht hervor, unter welchen Voraussetzung Preise für sich betrachtet unter den Begriff des Geschäftsgeheimnisses fallen können. Dies ist auf die eingeschränkte Kognition des Bundesgerichts zurückzuführen. Da es nur eine Willkürprüfung vorgenommen hat, kann nicht davon ausgegangen werden, dass Preise auch in Anwendung des Bundesrechts als Geschäftsgeheimnis im Sinne einer Ausnahme des Öffentlichkeitsprinzips qualifiziert werden würden. In Anwendung des basel-städtischen Rechts hat das Bundesgericht die erfolgte Interessenabwägung als für nicht willkürlich gewürdigt. Daher hat das Bundesgericht darauf verzichtet, vertiefte Überlegungen anzustellen, ob Preise für sich betrachtet Geschäftsgeheimnisse sind.

32 Durch den Ausschluss einer Interessenabwägung im Öffentlichkeitsgesetz des Bundes wäre der vorliegende Fall in Anwendung des Bundesrechts wohl anders entschieden worden. Nach Bundesrecht wären Preise vorliegend nicht als Geschäftsgeheimnisse qualifiziert worden und somit hätten sie wohl zugänglich gemacht werden müssen.

33 Demnach kann der Zugang zu einem Preis nach dem Öffentlichkeitsgesetz des Bundes nicht mit der Begründung verweigert werden, er sei ein Geschäftsgeheimnis im Sinne des Öffentlichkeitsrechts. Der Bundesgerichtsentscheid kann somit für die Beurteilung von Geschäftsgeheimnissen nach dem Öffentlichkeits- 
gesetz des Bundes keine präjudizielle

Wirkung entfalten.

sui-generis 2019, S. 226 